\title{
Inclusion of Mechanical Ventilation in Severity Staging of Tuberculous Meningitis Improves Outcome Prediction
}

\author{
Jayantee Kalita, ${ }^{1 *}$ Usha K. Misra, ${ }^{1}$ Varun K. Singh, ${ }^{2}$ Prakash C. Pandey, ${ }^{1}$ and Justin Thomas ${ }^{1}$ \\ ${ }^{1}$ Department of Neurology, Sanjay Gandhi Post Graduate Institute of Medical Sciences, Lucknow, India; ${ }^{2}$ Department of Neurology, Institute of \\ Medical Sciences, Banaras Hindu University, Varanasi, India
}

\begin{abstract}
Patients with tuberculous meningitis (TBM) in any stage of the British Medical Research Council (BMRC) scale, if requiring mechanical ventilation (MV), are likely to have a poor outcome. We report the usefulness of BMRC, BMRC-MV, and BMRC-hydrocephalus (BMRC-HC) staging, and Haydarpasa Meningitis Severity Index (HAMSI) scoring in predicting the outcome of TBM. One hundred ninety-seven TBM patients were analyzed from a prospectively maintained TBM registry. The severity of meningitis was categorized using BMRC (stages I-III), BMRC-MV (I-IV [MV patients were grouped as stage IV]), and BMRC-HC (I-IV [BMRC stage III patients with hydrocephalus were grouped as stage IV]). Haydarpasa Meningitis Severity Index scoring was categorized as $<6$ and $\geq 6$. The outcome was defined at 6 months using the modified Rankin Scale (mRS) as death, poor (mRS score $>2$ ), or good (mRS score $\leq 2)$. Forty-nine (25\%) patients died. BMRC-mechanical ventilation stage IV had the highest predictive value for defining death, with a sensitivity of $88 \%$ and a specificity of $86 \%$. About $81.7 \%$ of surviving patients had a good outcome at 6 months. BMRC-mechanical ventilation stages I-III had the highest predictive value for defining good outcome, with a sensitivity of $93 \%$ and a specificity of $61 \%$. In TBM, BMRC-MV staging has the best predictive value for defining death and disability.
\end{abstract}

\section{INTRODUCTION}

Tuberculosis is a major health problem in the developing countries. The annual incidence of tuberculosis in the developing countries is $110-165$ cases $/ 100,000$ and that in the United States is $9 / 100,000$ population. ${ }^{1}$ Central nervous system (CNS) tuberculosis results in $20-30 \%$ deaths, and up to $50 \%$ survivors have severe neurological sequelae. ${ }^{2-7}$ Tuberculous meningitis (TBM) has diverse clinical manifestations ranging from a mild illness to deep coma. Defining disease severity is important for treatment strategies and predicting outcome. The severity of TBM was defined in 1948 by the British Medical Research Council (BMRC) into stages I, II, and III based on meningitis symptoms, focal neurological deficit, and level of consciousness. ${ }^{8}$ In the earlier studies, the outcome of TBM was related to the stage of meningitis, but in the post-computerized tomography (CT) scan and magnetic resonance imaging (MRI) era, many studies did not find the relationship of BMRC with outcome. ${ }^{9-11}$ The discovery of CT scan and MRI has helped in understanding the structural brain changes in TBM, and these imaging techniques are greatly useful in the diagnosis and management. Hydrocephalus is although a correctable complication of TBM, but it predicts a poor outcome. ${ }^{12-14}$ Similarly, infarction is also a poor prognostic predictor of TBM. ${ }^{15}$ A study on TBM has reported better predictive value of the Haydarpasa Meningitis Severity Index (HAMSI) scoring system including clinical, radiological, and comorbidities than the BMRC scale. ${ }^{13}$ The development of neurocritical care provided opportunity to treat seriously ill TBM patients, who otherwise might have died. In a study, TBM patients requiring mechanical ventilation (MV) had a higher mortality $(73 \%)$ than those who did not require MV $(1.1 \%) .^{16}$ Similar results have also been reported by others. ${ }^{12,17}$ It may be appropriate to stage the patients requiring MV to a separate stage, which may have a better predictive value.

\footnotetext{
${ }^{*}$ Address correspondence to Jayantee Kalita, Department of Neurology, Sanjay Gandhi Post Graduate Institute of Medical Sciences, Lucknow 226014, India. E-mails: jayanteek@yahoo.com or jkalita@ sgpgi.ac.in
}

Similarly, categorizing the patients in the BMRC stage III with hydrocephalus to a different group may also improve the predictive value. In this study, we compare the relative usefulness of BMRC, BMRC-mechanical ventilation (BMRC-MV), BMRC-hydrocephalus (BMRC-HC), and HAMSI scoring in predicting death and disability of the patients with TBM.

Subjects and methods. This study was conducted in a tertiary care teaching hospital in India. The patients with TBM admitted during January 2014-December 2018 were included from a prospectively maintained TBM registry, which was approved by the Institute Ethics Committee (2013-83-EMP72; 2018/165PGI-BE).

Inclusion criteria. The diagnosis of TBM was based on clinical, cerebrospinal fluid (CSF), and CT/MRI criteria. $^{5}$

1. Essential criteria: This group included patients with fever, headache, and/or vomiting for more than 5 days in whom malaria, septic, and fungal meningitis were excluded.

2. Supportive criteria:

a. Cerebrospinal fluid cells $10-500 / \mathrm{mm}^{3}$ (> 50\% lymphocytes), protein $>1 \mathrm{gm} / \mathrm{l}$, and negative culture for pyrogenic bacteria and fungi;

b. Cranial CT or MRI evidences of exudates, hydrocephalus, infarction, or tuberculoma in isolation or various combinations; and

c. Evidence of extra CNS tuberculosis.

3. Exclusion of alternative diagnosis.

Exclusion criteria. The patients with organ transplantation, malignancy, pregnancy, preexisting CNS disease, immunemediated disease, preexisting liver disease, and kidney failure and those on immunosuppressant or chemotherapy were excluded.

Clinical evaluation. A detailed clinical history including demographic information and comorbidities was noted. The level of consciousness was assessed by the Glasgow Coma Scale (GCS). The presence of papilledema, cranial nerve palsy, muscle weakness, and abnormality in tone and tendon reflexes was noted. Sensations and coordination were tested in conscious cooperative patients. 
Investigations. Complete hemogram, serum chemistry, chest radiograph, ultrasound abdomen, and electrocardiography were performed. Cerebrospinal fluid was analyzed for cells, protein, and glucose. Cerebrospinal fluid was also subjected to smear and culture for Mycobacterium tuberculosis and pyogenic bacteria. Polymerase chain reaction (PCR) for $M$. tuberculosis and enzyme-linked immunosorbent assay for cryptococcal antigen in CSF were also performed.

Cranial imaging. Cranial CT scan was performed using a third-generation CT scanner, and cranial MRI was performed using a 3T MRI scanner (GE medical system, Milwaukee, WI). Axial images were obtained in T1, T2, fluid attenuation inversion recovery (FLAIR), diffusion weighted image (DWI), and T1 contrast. The presence of exudates, hydrocephalus, infarction, and tuberculoma was noted.

Diagnostic categorization of TBM. The patients were diagnosed as definite TBM if acid-fast bacillus was demonstrated in smear or culture, or if PCR was positive for M. tuberculosis. In the absence of bacterial confirmation, patients with essential and two supportive criteria were categorized as probable TBM and essential with one supportive criterion as possible TBM. ${ }^{4}$

Severity staging of TBM. The patients were categorized using the following staging criteria:

British Medical Research Council staging. Stage I = meningitis only, stage $\|=$ meningitis with lethargy or cranial nerve palsy, and stage III = meningitis with stupor, gross paralysis, or paresis. ${ }^{8}$ In stage III, we considered GCS score $<11$, and for stage II GCS scores 11-14 for quantification of altered sensorium.

British Medical Research Council-mechanical ventilation staging. Stages I-III as before. Any patient in stage I, II, or III requiring MV was categorized as stage IV.

British Medical Research Council-hydrocephalus staging. Stages I-III as before. Patients in stage III having hydrocephalus on CT or MRI were categorized as stage IV.

Haydarpasa Meningitis Severity Index scoring. It is a composite scoring in which altered sensorium weighted as 3 , altered sensorium with nausea and vomiting 1 , diabetes mellitus 3 , immunosuppression 2 , neurological deficit 1 , hydrocephalus 1 , and vasculitis $1 .{ }^{13}$ We have considered vasculitis if there was infarction on MRI.

The BMRC staging was performed at admission, and the others were performed at the worst clinical and/or radiological condition during the hospital stay.

Treatment. All the patients received standard fourdrug antitubercular treatment (isoniazide $5 \mathrm{mg} / \mathrm{kg}$ to $300 \mathrm{mg}$, rifampicin $10 \mathrm{mg} / \mathrm{kg}$ to $450 \mathrm{mg}$, ethambutol $15 \mathrm{mg} / \mathrm{kg}$ to $800 \mathrm{mg}$, and pyrazinamide $25 \mathrm{mg} / \mathrm{kg}$ to $1,500 \mathrm{mg}$ daily). ${ }^{18}$ Some patients also received levofloxacin $500 \mathrm{mg}$ daily. All the patients received prednisolone $40 \mathrm{mg}$ daily for 1 month, which was then tapered in the next month. Aspirin $150 \mathrm{mg}$ was also prescribed to all. The patients with seizure received levetiracetam, clobazam, or lacosamide, and those with hydrocephalus with deteriorating consciousness were treated with ventriculoperitoneal shunt surgery. The patients with respiratory failure evidenced by acidosis $(\mathrm{pH}<7.3)$, hypercarbia $\left(\mathrm{PaCO}_{2}>50 \mathrm{~mm}\right.$ of $\left.\mathrm{Hg}\right)$, or hypoxemia $\left(\mathrm{PaO}_{2}<60 \mathrm{~mm}\right.$ of $\left.\mathrm{Hg}\right)$ in arterial blood gas analysis (ABG) were intubated and mechanically ventilated. ${ }^{16}$ They were monitored in the intensive care unit (ICU) and ABG, blood counts, and blood or body fluid cultures were performed as and when needed. All the patients received supportive care including fluid, electrolyte, calorie, and nursing care.

Outcome. The patients were followed up for 6 months. Day of death and good outcome from the initiation of treatment was noted. At 6 months, the outcome was assessed by the modified Rankin Scale (mRS), ${ }^{19}$ and was categorized as good $(m R S \leq 2)$ or poor $(m R S>2){ }^{20}$

Statistical analysis. Death and poor outcome were compared among BMRC, BMRC-MV, BMRC-HC staging, and HAMSI scoring. Sensitivity, specificity, positive predictive value, and negative predictive value in predicting death and poor outcome at 6 months of three different staging systems were evaluated. Kaplan-Meier analysis was performed for cumulative survival using BMRC, BMRC$\mathrm{MV}$, BMRC-HC staging, and HAMSI scoring system. A similar statistical analysis was also performed for poor outcome. Univariate regression analysis was performed for death and poor outcome using clinical, laboratory, and MRI findings followed by multivariate analysis with Bonferroni correction. A hazard ratio of various stagings in predicting death and poor outcome was also evaluated using Cox regression analysis. A corresponding "test statistic" having a two-tailed $P$-value of $<0.05$ was considered significant. The statistical analysis was performed using SPSS 20 version (Chicago, IL).

\section{RESULTS}

One-hundred and ninety-seven patients with TBM were included. The median age was 30 (range 3-75) years, 103 $(52 \%)$ of whom were males. Sixty $(30 \%)$ patients had definite TBM, and the remaining $137(70 \%)$ had probable TBM. Cranial MRI was abnormal in 177 (90\%) patients and included exudates in 100 (51\%), hydrocephalus in 89 (45\%), tuberculoma in 115 (58\%), and infarctions in 76 (39\%) patients. The clinical and radiological details are presented in Supplemental Table 1. The proportions of patients in four staging scales are summarized in Table 1. Three patients from BMRC stage 1 and 30 patients each from BMRC stages II and III required MV

TABLE 1

Proportion of tuberculous meningitis patients in different severity staging scales

\begin{tabular}{ll}
\hline BMRC staging, $n(\%)$ & $39(20)$ \\
I & \\
II & $94(48)$ \\
III & $64(32)$ \\
BMRC-mechanical ventilation staging, $n(\%)$ & \\
I & $36(18)$ \\
I & $64(32)$ \\
III & $34(17)$ \\
IV & $63(32)$ \\
BMRC-hydrocephalus staging, $n(\%)$ & \\
I & $39(20)$ \\
II & $94(48)$ \\
III & $31(16)$ \\
IV & $33(16)$ \\
Haydarpasa Meningitis Severity Index score, $n(\%)$ & \\
$\leq 1$ & $42(21)$ \\
2 & $25(13)$ \\
3 & $29(15)$ \\
4 & $43(22)$ \\
5 & $33(16)$ \\
$\geq 6$ & $25(13)$ \\
\hline BMRC British Medical Research Council
\end{tabular}

$\mathrm{BMRC}=$ British Medical Research Council 
during the hospital stay, and hence these patients were categorized as stage IV in the BMRC-MV staging. Thirty-three patients from BMRC stage III developed hydrocephalous and were categorized as BMRC-HC stage IV.

Association of death with various severity staging scales. Forty-nine (25\%) patients died; 21 (43\%) within 1 month, 24 (49\%) during 1-3 months, and four (8\%) during 3-6 months of antitubercular treatment. The proportions of deaths in BMRC stage II (24/49, 49\%) and stage III (20/49, $41 \%)$ were higher than those of deaths in stage I (5/49, 10\%). In BMRC-MV staging, 43 (88\%) deaths occurred in stage IV patients. In BMRC-HC staging, eight (16\%) patients died in stage IV. The occurrence of death was not proportional to the HAMSI score. BMRC-mechanical ventilation stage IV had the highest predictive value for death, with a sensitivity of $88 \%$ and a specificity of $86 \%$ followed by BMRC stage III, $41 \%$ and $70 \%$, respectively (Supplemental Table 2, Table 2). On the Kaplan-Meier survival analysis, there was no difference in the survival times of the patients in stages I, II, and III of the BMRC staging $(P=0.11)$. On the BMRC-MV scale, there was a significant difference in the proportion of patients in stages I, II, III, and IV. The log-rank test showed a significant difference in the survival time between stage IV and other three stages $(P<$ $0.001)$; but there was no difference between stages I-III. The BMRC-HC scale did not show a significant difference between the stages $(P=0.09)$. In the HAMSI scoring, we had observed a higher probability of survival in the patients who scored 6 or more $(P=0.009$; Figure 1$)$.
Functional outcome at 6 months. At 6 months, 121/148 patients $(81.7 \%)$ had a good outcome. The predictive value of good outcome in the surviving patients was the highest in the BMRC-MV staging, with a sensitivity of $93 \%$ and specificity of $61 \%$ in stages I, II, and III patients. The sensitivity of BMRC stages I and II in predicting a good outcome was $75 \%$, with a specificity of $41 \%$. The BMRC-HC staging had a higher sensitivity with a lower specificity. The details are presented in Table 3 and Supplemental Table 2. On the Kaplan-Meier analysis, stage IV of the BMRC-MV scale had the least probability of a good outcome compared with stages I-III. On the BMRC scale, there was also a trend of worse outcome with increasing severity. The increasing severity of meningitis in the BMRC-HC and HAMSI score did not follow any trend (Figure 2). On univariate analysis, BMRC stage III $(P=0.01)$, BMRC-MV stage IV $(P<0.001)$, BMRC-HC stage IV $(P<0.02)$, HAMSI score $(P=0.003)$, status epilepticus $(P=0.007)$, and duration of illness $(P=$ $0.02)$ were associated with poor outcome. On Cox regression analysis, poor outcome was also less frequent in BMRC-MV stage I (adjusted hazard ratio [AHR] 0.039; 95\% Cl 0.004-0.39; $P=0.006$ ), stage II (AHR 0.161; 95\% Cl $0.075-0.347 ; P<0.001$ ), and stage III (AHR 0.215; $95 \% \mathrm{CI}$ $0.086-0.541 ; P=0.001)$ than in stage IV. The BMRC-HC did not follow any trend.

Predictors of death and disability using baseline parameters including BMRC. At admission, 39 (20\%) patients were in stage I, 94 (48\%) in stage II, and 64 (32\%) in stage III on

TABLE 2

Relationship of various clinical and radiological variables with death in patients with tuberculous meningitis

\begin{tabular}{|c|c|c|c|}
\hline & Survived $(n=148)$ & Death $(n=49)$ & $P$-value \\
\hline Age (years) & $32.95 \pm 15.95$ & $32.06 \pm 12.06$ & 0.72 \\
\hline Gender (female), $n$ (\%) & $71(48)$ & $23(47)$ & 0.90 \\
\hline Duration of illness (days) & $60(5-210)$ & $60(7-240)$ & 0.19 \\
\hline Glasgow Coma Scale score & $12.20 \pm 2.95$ & $10.84 \pm 3.46$ & 0.01 \\
\hline Cranial nerve palsy, $n(\%)$ & $58(39)$ & $13(26)$ & 0.11 \\
\hline Focal motor deficit, $n(\%)$ & $87(59)$ & 29 (59) & 0.96 \\
\hline Seizure, $n(\%)$ & $63(42)$ & $34(69)$ & 0.001 \\
\hline \multicolumn{4}{|l|}{ Magnetic resonance imaging, $n(\%)$} \\
\hline Exudate & $76(51)$ & $24(49)$ & 0.77 \\
\hline Hydrocephalus & $67(45)$ & $22(45)$ & 0.96 \\
\hline Infarction & $59(40)$ & $17(35)$ & 0.52 \\
\hline Tuberculoma & $90(61)$ & $25(51)$ & 0.23 \\
\hline BMRC staging, $n(\%)$ & & & 0.11 \\
\hline I & $34(23)$ & $5(10)$ & \\
\hline II & $70(47)$ & $24(49)$ & \\
\hline III & $44(30)$ & $20(41)$ & \\
\hline BMRC-mechanical ventilation staging, $n(\%)$ & & & $<0.001$ \\
\hline 1 & $34(28)$ & $2(4)$ & \\
\hline II & $61(41)$ & $3(6)$ & \\
\hline III & $33(22)$ & $1(2)$ & \\
\hline IV (any stage + MV) & $20(13.5)$ & $43(88)$ & \\
\hline BMRC-hydrocephalus staging, $n$ (\%) & & & 0.10 \\
\hline I & $34(23)$ & $5(10)$ & \\
\hline ॥ & $70(47)$ & $24(49)$ & \\
\hline III & 19 (13) & $12(25)$ & \\
\hline IV (stage III + HC) & $25(17)$ & $8(16)$ & \\
\hline $\begin{array}{l}\text { Haydarpasa Meningitis Severity Index } \\
\text { score, } n(\%)\end{array}$ & & & 0.01 \\
\hline$\leq 1$ & $37(25)$ & $5(10)$ & \\
\hline 2 & $14(9)$ & $11(22)$ & \\
\hline 3 & $22(15)$ & $7(14)$ & \\
\hline 4 & $31(21)$ & $12(25)$ & \\
\hline 5 & $21(14)$ & $12(25)$ & \\
\hline$\geq 6$ & $23(16)$ & $2(4)$ & \\
\hline
\end{tabular}

BMRC $=$ British Medical Research Council. 

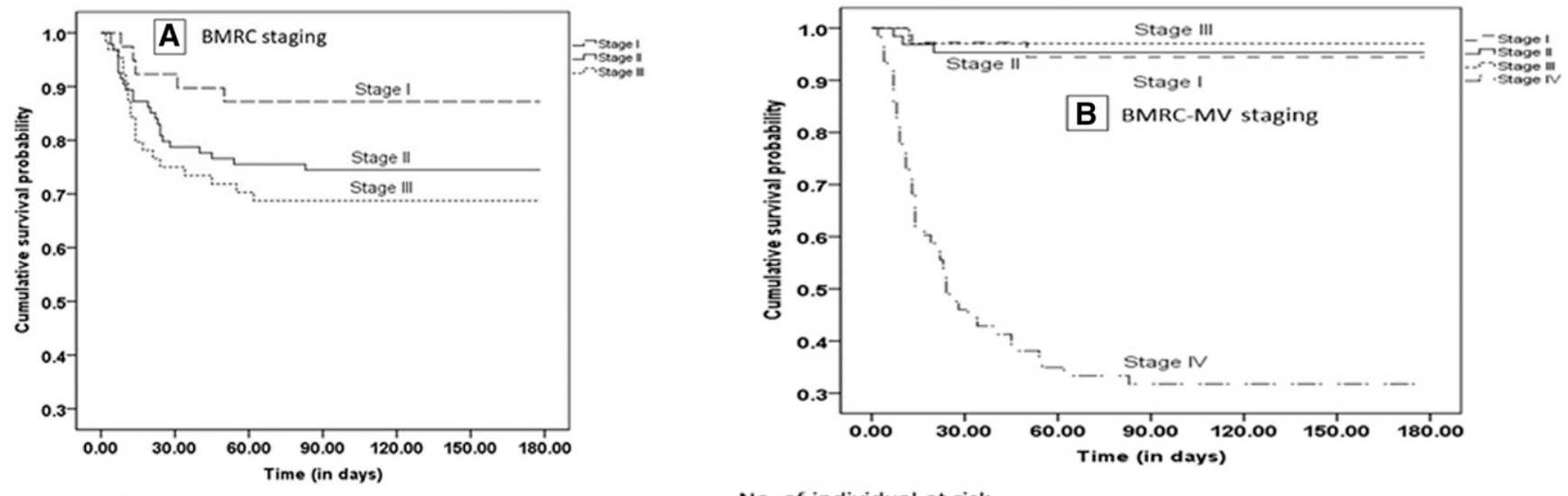

\begin{tabular}{llllllll}
\multicolumn{7}{l}{ Number of individuals at risk } \\
Stage I & 39 & 36 & 34 & 34 & 34 & 34 & 34 \\
Stage II & 94 & 74 & 71 & 70 & 70 & 70 & 70 \\
Stage III & 64 & 48 & 45 & 44 & 44 & 44 & 44
\end{tabular}

\begin{tabular}{lccccccc}
\multicolumn{9}{l}{$\begin{array}{l}\text { No. of individual at risk } \\
\text { Stage I }\end{array}$} & 36 & 35 & 34 & 34 & 34 & 34 & 34 \\
Stage II & 64 & 61 & 61 & 61 & 61 & 61 & 61 \\
Stage III & 34 & 33 & 33 & 33 & 33 & 33 & 33 \\
Stage IV & 63 & 29 & 22 & 20 & 20 & 20 & 20
\end{tabular}
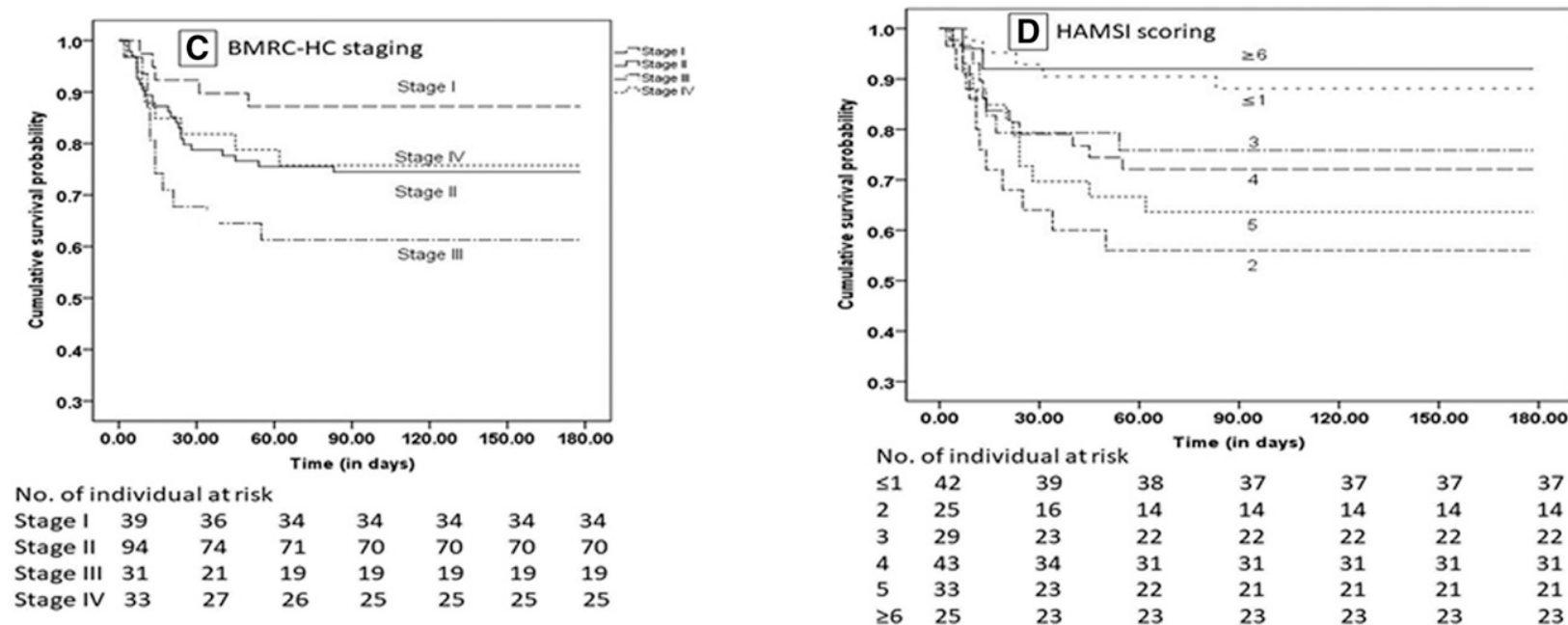

$\begin{array}{llllllll}\text { No. of individual at risk } \\ \text { Stage I } & 39 & 36 & 34 & 34 & 34 & 34 & 34 \\ \text { Stage II } & 94 & 74 & 71 & 70 & 70 & 70 & 70 \\ \text { Stage III } & 31 & 21 & 19 & 19 & 19 & 19 & 19 \\ \text { Stage IV } & 33 & 27 & 26 & 25 & 25 & 25 & 25\end{array}$

FIGURE 1. Kaplan-Meier survival curves show cumulative survival probability in (A) British Medical Research Council (BMRC) scale, (B) BMRCmechanical ventilation (BMRC-MV) scale, (C) BMRC-hydrocephalus scale, and (D) Haydarpasa Meningitis Severity Index (HAMSI) score. The logrank test shows that there is no difference in the survival time of the patients between three stages of BMRC $(P=0.11)$ and four stages of BMRC-HC $(P=0.09)$. There is a significant difference in survival time between stage IV and other three stages of BMRC-MV $(P=<0.001)$, but for the rest of the pairs, it is statistically insignificant. The difference is also significant in the survival time among the patients with different $\mathrm{HAMSI}$ scores $(P=0.009)$, but there is no increase of deaths with rise in score. The chart above the graphs shows the number of individuals at risk.

the BMRC staging. On univariate analysis, survival at 6 months was related to GCS score $(P=0.007)$ and seizure $(P=0.002)$. On Cox regression analysis also, the independent predictors of 6-month survival were GCS score (AHR 0.91; 95\% Cl $0.84-0.99 ; P=0.03$ ) and seizure (AHR 2.38; 95\% Cl 1.28-4.40; $P=0.006)$.

Outcome predictors in mechanically ventilated patients. Sixty-three patients needed MV, of whom 43 (68.2\%) died and $12(19 \%)$ only had a good outcome. The clinical features, MRI findings, and comorbidities in the patients who died or had poor recovery were similar to those who survived or had a good outcome (Supplemental Tables 3 and 4).

\section{DISCUSSION}

In the present study, the BMRC-MV staging in TBM had the highest predictive value for defining death and disability at 6 months compared with the BMRC staging. The BMRC-HC staging and the HAMSI scoring did not have a linear relationship with death and functional outcome. The need of MV in any disease suggests more severe illness with higher mortality. ${ }^{21-23}$ Patients with septicemia, H1N1, dengue and scrub typhus on the MV have fewer deaths and good functional outcomes unlike TBM. ${ }^{21,24,25}$ In a study on 164 patients with acute encephalitis syndrome (AES), 69 (42\%) needed MV. Forty-three (26\%) patients died, and all were in the MV group. Higher Sequential Organ Failure Assessment score and untreatable etiology were independent predictors of death. However, at 3 months, 14\% of patients had poor outcome. In this study, the etiologies of AES patients were viral in $44(27 \%)$, scrub typhus in 48 (29\%), malaria in six (4\%), and unknown in $56(34 \%) .{ }^{21}$ In a retrospective study on 103 patients with encephalitis illness of various etiology (viral in $27.2 \%$, bacterial and fungal in $9.7 \%$, autoimmune in $16.5 \%$, and undetermined in $46.6 \%$ ), about $95 \%$ of mechanically ventilated patients died. $^{22}$ In another retrospective study including 198 patients with AES, MV, age $\geq 65$ years, immunocompromised states, thrombocytopenia, and CSF polymorphonuclear pleocytosis 
TABLE 3

Relationship of various clinical and radiological variables with functional outcome at 6 months in the surviving patients with tuberculous meningitis

\begin{tabular}{|c|c|c|c|}
\hline & Good $(n=121)$ & Poor $(n=27)$ & $P$-value \\
\hline Age (years) & $32.84 \pm 16.2$ & $33.4 \pm 15$ & 0.86 \\
\hline Gender (female), $n$ (\%) & $60(49.5)$ & $11(41)$ & 0.40 \\
\hline Duration of illness (days) & $66.92 \pm 59.07$ & $92.25 \pm 57.89$ & 0.045 \\
\hline Glasgow Coma Scale score & $12.5 \pm 2.9$ & $10.8 \pm 2.9$ & 0.007 \\
\hline Cranial nerve palsy, $n(\%)$ & $43(35)$ & $15(56)$ & 0.054 \\
\hline Focal motor deficit, $n$ (\%) & $65(54)$ & $22(81)$ & 0.008 \\
\hline Seizure, $n(\%)$ & $48(40)$ & $15(56)$ & 0.13 \\
\hline Status epilepticus, $n$ (\%) & $5 / 48(10)$ & $5 / 15(33)$ & 0.049 \\
\hline \multicolumn{4}{|l|}{ Magnetic resonance imaging, $n(\%)$} \\
\hline Exudate & $58(48)$ & $18(67)$ & 0.08 \\
\hline Hydrocephalus & $49(40)$ & $18(67)$ & 0.01 \\
\hline Infarction & $44(36)$ & $15(56)$ & 0.06 \\
\hline Tuberculoma & $70(58)$ & $20(74)$ & 0.12 \\
\hline BMRC staging, $n(\%)$ & & & 0.006 \\
\hline 1 & $34(28)$ & 0 & \\
\hline II & $52(43)$ & $18(67)$ & \\
\hline III & $35(29)$ & $9(33)$ & \\
\hline BMRC-mechanical ventilation staging, $n(\%)$ & & & 0.001 \\
\hline II & $34(28)$ & 0 & \\
\hline II & $48(40)$ & $13(48)$ & \\
\hline III & 27 (22) & $6(22)$ & \\
\hline IV (any stage + MV) & $12(10)$ & $8(30)$ & \\
\hline BMRC-hydrocephalus staging, $n$ (\%) & & & 0.003 \\
\hline I & $34(28)$ & 0 & \\
\hline ॥ & $52(43)$ & $18(67)$ & \\
\hline III & $15(12)$ & $4(15)$ & \\
\hline IV (stage III + HC) & $20(17)$ & 5 (18.5) & \\
\hline Haydarpasa Meningitis Severity Index & & & 0.002 \\
\hline \multicolumn{4}{|l|}{ Severity score, $n(\%)$} \\
\hline$\leq 1$ & $36(30)$ & $1(3.7)$ & \\
\hline 2 & $13(11)$ & $1(3.7)$ & \\
\hline 3 & 19 (15) & $3(11)$ & \\
\hline 4 & $24(20)$ & $7(26)$ & \\
\hline 5 & $12(10)$ & 9 (33) & \\
\hline$\geq 6$ & $17(14)$ & $6(22)$ & \\
\hline
\end{tabular}

$\mathrm{BMRC}=$ British Medical Research Council.

were associated with poor prognosis. ${ }^{23}$ Another study compared death and functional outcome in $38 \mathrm{MV}$ and 36 non-MV patients with TBM. About $71 \%$ of MV patients died, and the remaining patients had poor outcome at 3 months, whereas none in the non-MV group died, and only $11 \%$ patients had a poor outcome. ${ }^{16}$ This study raised the possibility of grouping the MV patients of any BMRC stage to a separate stage.

Various staging scales have been described to define the severity of TBM to predict death and disability ${ }^{8,9,13}$ BMRC staging is mainly used in most of the studies and has been reported as a robust predictor of short- and long-term outcomes. ${ }^{8,26-31}$ However, in the era of intensive care facilities, many authors did not find the BMRC staging as a prognostic predictor. ${ }^{9,13,32}$ In the initial BMRC staging, altered sensorium was measured subjectively as the GCS score was developed in $1974 .^{33}$ Since then, the GCS score has been an independent predictor of outcome. ${ }^{34-38}$ Therefore, we have used the GCS score for defining various grades of altered sensorium in BMRC staging. The BMRC staging was able to define patients with good outcomes at 6 months between stages I-III meningitis, but there was no difference in deaths among the three groups. Using the BMRC-MV staging, patients in stage IV stratified the highest deaths and the worst outcome compared with the patients in stages I-III, but there was no difference between the stages I-III. In a study on 43
TBM patients, the Acute Physiology and Chronic Health Evaluation II and the GCS score were more sensitive than the BMRC staging in predicting the death and disability. ${ }^{9}$ In our study, there was no linear correlation of the HAMSI score with death and disability. This may be because of the less number of patients with immunosuppression and diabetes in our cohort.

The higher proportion of deaths in TBM patients needing MV in our study is unlikely because of the quality of ICU care because mortality in Guillain-Barre syndrome and myasthenic crisis in our ICU are comparable to the developed countries. ${ }^{39-41}$ Higher deaths in the TBM patients compared with dengue, scrub typhus, and Japanese encephalitis may be due to the difference in underlying disease pathophysiology. Dengue and Japanese encephalitis are monophasic illness; once the acute crisis is over, the patients start improving. Similarly, cerebral malaria and scrub typhus respond to the specific treatment. Although tuberculous meningitis is a treatable disease, it requires longer duration of treatment and about $40-60 \%$ may deteriorate on antitubercular treatment because of paradoxical response. ${ }^{42}$

Limitation. This is a retrospective study, although the data were obtained from a prospectively maintained registry. Ours is a referral center, and more severe meningitis dominated the study cohort. The application of this BMRC-MV in the non-ICU setting may be extrapolated to any BMRC patients with respiratory failure based on clinical and ABG parameters. 

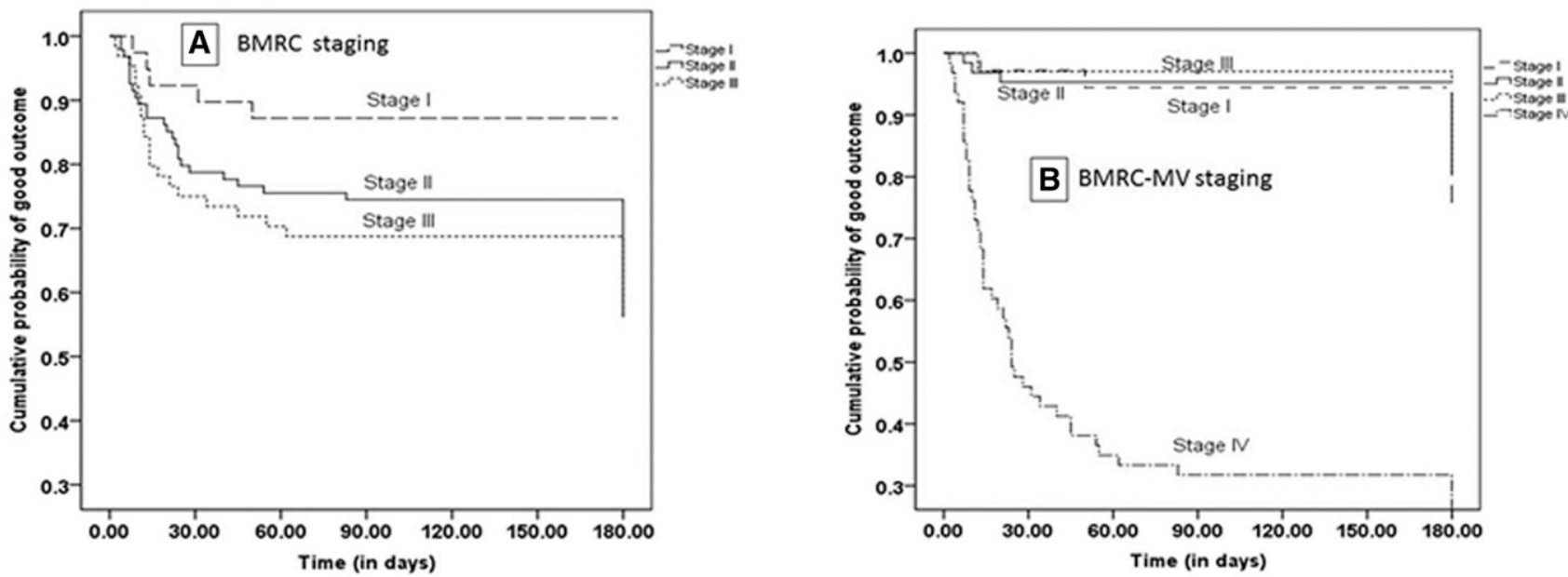

\begin{tabular}{|c|c|c|c|c|c|c|c|}
\hline \multicolumn{8}{|c|}{ No. of individual at risk } \\
\hline Stage I & 39 & 36 & 34 & 34 & 34 & 34 & 3 \\
\hline Stage II & 94 & 74 & 71 & 70 & 70 & 70 & 52 \\
\hline Stage III & 64 & 48 & 45 & 44 & 44 & 44 & 35 \\
\hline
\end{tabular}

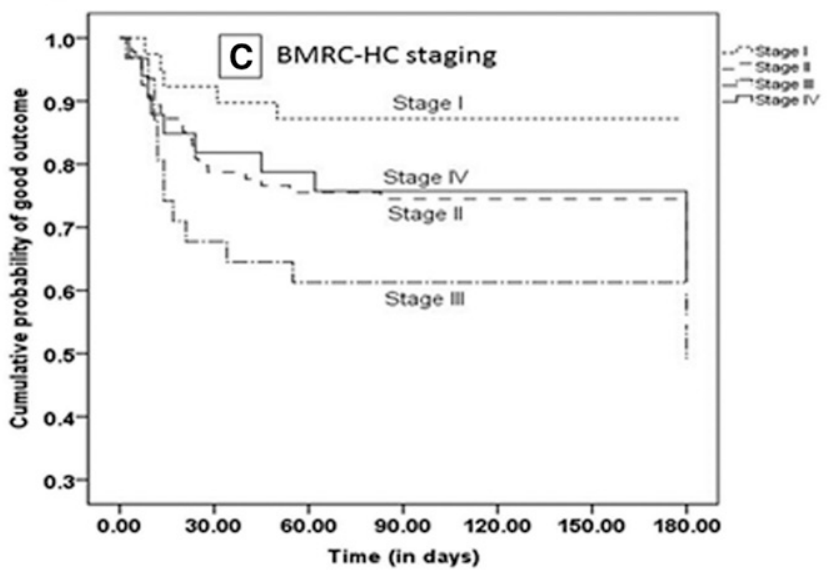

No. of individual at risk

$\begin{array}{llllllll}\text { Stage I } & 39 & 36 & 34 & 34 & 34 & 34 & 34 \\ \text { Stage II } & 94 & 74 & 71 & 70 & 70 & 70 & 52 \\ \text { Stage III } & 31 & 21 & 19 & 19 & 19 & 19 & 15 \\ \text { Stage IV } & 33 & 27 & 26 & 25 & 25 & 25 & 20\end{array}$

$\begin{array}{llllllll}\text { No. of individual at risk } & & & & & \\ \text { Stage I } & 36 & 35 & 34 & 34 & 34 & 34 & 34 \\ \text { Stage II } & 64 & 61 & 61 & 61 & 61 & 61 & 48 \\ \text { Stage III } & 34 & 33 & 33 & 33 & 33 & 33 & 27 \\ \text { Stage IV } & 63 & 29 & 22 & 20 & 20 & 20 & 12\end{array}$

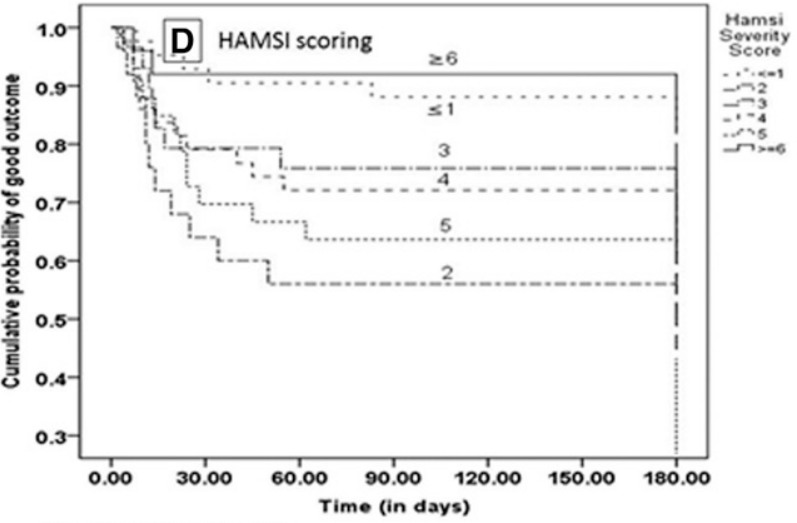

\begin{tabular}{llllllll}
\multicolumn{7}{l}{ No. of individual at risk } \\
$\leqslant 1$ & 42 & 39 & 38 & 37 & 37 & 37 & 34 \\
2 & 25 & 16 & 14 & 14 & 14 & 14 & 12 \\
3 & 29 & 23 & 22 & 22 & 22 & 22 & 17 \\
4 & 43 & 34 & 31 & 31 & 31 & 31 & 23 \\
5 & 33 & 23 & 22 & 21 & 21 & 21 & 8 \\
$\geq 6$ & 25 & 23 & 23 & 23 & 23 & 23 & 11
\end{tabular}

FiguRE 2. Kaplan-Meier survival curves show difference in good outcome during 6 months of follow-up in the patients with tuberculous meningitis in (A) British Medical Research Council (BMRC) scale, (B) BMRC-mechanical ventilation (BMRC-MV) scale, (C) BMRC-hydrocephalus scale, and Haydarpasa Meningitis Severity Index (HAMSI) score of $\leq 1,2,3,4,5$, and $\geq 6$ (D). The log-rank test shows that there is a significant difference in the probability of good outcomes among three stages of BMRC $(P=0.003)$, and between stage IV and other three stages of BMRC-MV $(P<0.001)$. The log-rank test also shows that the difference in the probabilities of good outcome among the different stages of BMRC-HC is significant, except in stages II and IV $(P=0.005)$. However there is no inverse relationship of good outcome with the HAMSI score. The chart below the graphs shows the number of individuals at risk. The patients with poor outcome group also included death in the analysis.

Conclusion. The BMRC-MV staging in TBM has the best predictive value in defining death and functional outcome at 6 months. Future studies in TBM are desirable, aiming at deriving necessary treatment strategies in MV patients.

Received January 29, 2020. Accepted for publication April 15, 2020. Published online May 26, 2020.

Note: Supplemental tables appear at www.ajtmh.org.

Acknowledgments: We thank Dr. Prabhaker Mishra, assistant professor of Biostatistics, Sanjay Gandhi PGI, for his statistical analysis and Mr. Shakti Kumar for his secretarial help. The American Society of Tropical Medicine and Hygiene (ASTMH) assisted with publication expenses.
Financial support: This study was partly supported by the Indian Council of Medical Research, government of India. One junior research fellow and a technical staff were appointed for collection of sample, analysis, and data entry.

Disclosure: This study was approved by the Institute Ethics Committee (2013-83-EMP-72; 2018/165PGI-BE), SGPGIMS, Lucknow, India. All the procedures performed in studies involving human participants were in accordance with the ethical standards of the institutional and /or national research committee and with the 1964 Helsinki declaration and its later amendment or comparable ethical standards. Statistical analysis was conducted by Dr. V. K. S.

Authors' addresses: Jayantee Kalita, Usha K. Misra, Prakash C. Pandey, and Justin Thomas, Department of Neurology, Sanjay Gandhi Post Graduate Institute of Medical Sciences, Lucknow, India, E-mails: 
jayanteek@yahoo.com,drukmisra@rediffmail.com, drprakashpandey@ gmail.com, and dr.justinthomas@gmail.com. Varun K. Singh, Department of Neurology, Institute of Medical Sciences, Banaras Hindu University, Varanasi, India, E-mail: mailurvarun@gmail.com.

\section{REFERENCES}

1. Raviglione MC, Snider DE Jr., Kochi A, 1995. Global epidemiology of tuberculosis. Morbidity and mortality of a worldwide epidemic. JAMA 273: 220-226.

2. Misra UK, Kalita J, Maurya PK, 2011. Stroke in tuberculous meningitis. J Neurol Sci 303: 22-30.

3. Kalita J, Misra UK, Ranjan P, 2007. Predictors of long-term neurological sequelae of tuberculous meningitis: a multivariate analysis. Eur J Neurol 14: 33-37. Erratum in: Eur J Neurol 14: 357.

4. Kalita J, Misra UK, Prasad S, Bhoi SK, 2014. Safety and efficacy of levofloxacin versus rifampicin in tuberculous meningitis: an open-label randomized controlled trial. J Antimicrob Chemother 69: 2246-2251.

5. Marais S, Thwaites G, Schoeman JF, Török ME, Misra UK, Prasad K, Donald PR, Wilkinson RJ, Marais BJ, 2010. Tuberculous meningitis: a uniform case definition for use in clinical research. Lancet Infect Dis 10: 803-812.

6. Thwaites GE, Hie TT, 2005. Tuberculous meningitis: many questions, too few answers. Lancet Neurol 4: 160-170.

7. Lee HG, William T, Menon J, Ralph AP, Ooi EE, Hou Y, Sessions O, Yeo TW, 2016. Tuberculous meningitis is a major cause of mortality and morbidity in adults with central nervous system infections in Kota Kinabalu, Sabah, Malaysia: an observational study. BMC Infect Dis 16: 296.

8. Medical Research Council, 1948. Streptomycin treatment of tuberculous meningitis. Lancet 1: 582-596.

9. Chou CH, Lin GM, Ku CH, Chang FY, 2010. Comparison of the APACHE II, GCS and MRC scores in predicting outcomes in patients with tuberculous meningitis. Int J Tuberc Lung Dis 14: 86-92.

10. Tan EK, Chee MW, Chan LL, Lee YL, 1999. Culture positive tuberculous meningitis: clinical indicators of poor prognosis. Clin Neurol Neurosurg 101: 157-160.

11. Yasar KK, Pehlivanoglu F, Sengoz G, 2010. Predictors of mortality in tuberculous meningitis: a multivariate analysis of 160 cases. Int J Tuberc Lung Dis 14: 1330-1335.

12. Cantier M et al.; ENCEPHALITICA Study Group, 2018. Functional outcomes in adults with tuberculous meningitis admitted to the ICU: a multicenter cohort study. Crit Care 22: 210.

13. Erdem $\mathrm{H}$ et al., 2015. Hamsi scoring in the prediction of unfavorable outcomes from tuberculous meningitis: results of Haydarpasa-II study. J Neurol 262: 890-898.

14. Garg RK, Raut T, Malhotra HS, Jain A, 2013. Tuberculous meningitis and hydrocephalus. J Infect 66: 541-542.

15. Kalita J, Misra UK, Nair PP, 2009. Predictors of stroke and its significance in the outcome of tuberculous meningitis. J Stroke Cerebrovasc Dis 18: 251-258.

16. Misra UK, Kalita J, Betai S, Bhoi SK, 2015. Outcome of tuberculous meningitis patients requiring mechanical ventilation. $J$ Crit Care 30: 1365-1369.

17. Verdon R, Chevret S, Laissy JP, Wolff M, 1996. Tuberculous meningitis in adults: review of 48 cases. Clin Infect Dis 22: 982-988.

18. WHO, 2010. Treatment of Tuberculosis: Guidelines, 4th Edition. Geneva, Switzerland: World Health Organization.

19. Farrell B, Godwin J, Richards S, Warlow C, 1991. The United Kingdom transient ischaemic attack (UK-TIA) aspirin trial: final results. J Neurol Neurosurg Psychiatry 54: 1044-1054.

20. Misra UK, Kalita J, Kumar M, 2018. Safety and efficacy of fludrocortisone in the treatment of cerebral salt wasting in patients with tuberculous meningitis: a randomized clinical trial. JAMA
Neurol 75: 1383-1391. Erratum in: JAMA Neurol 2018;75(11): 1443.

21. Kalita J, Mani VE, Bhoi SK, Misra UK, 2017. Spectrum and outcome of acute infectious encephalitis/encephalopathy in an intensive care unit from India. QJM 110: 141-148.

22. Thakur KT, Motta M, Asemota AO, Kirsch HL, Benavides DR, Schneider EB, McArthur JC, Geocadin RG, Venkatesan A, 2013. Predictors of outcome in acute encephalitis. Neurology 81: 793-800.

23. Singh TD, Fugate JE, Rabinstein AA, 2015. The spectrum of acute encephalitis: causes, management, and predictors of outcome. Neurology 84: 359-366.

24. Zampieri FG, Mazza B, 2017. Mechanical ventilation in sepsis: a reappraisal. Shock 47(1S Suppl 1): 41-46.

25. Ríos FG et al., 2011. Lung function and organ dysfunctions in 178 patients requiring mechanical ventilation during the 2009 influenza A (H1N1) pandemic. Crit Care 15: R201.

26. Thwaites GE et al., 2004. Dexamethasone for the treatment of tuberculous meningitis in adolescents and adults. N Engl J Med 351: 1741-1751.

27. Hsu PC, Yang CC, Ye JJ, Huang PY, Chiang PC, Lee MH, 2010. Prognostic factors of tuberculous meningitis in adults: a 6-year retrospective study at a tertiary hospital in northern Taiwan. $\checkmark$ Microbiol Immunol Infect 43: 111-118.

28. Kalita J, Misra UK, 1999. Outcome of tuberculous meningitis at 6 and 12 months: a multiple regression analysis. Int $J$ Tuberc Lung Dis 3: 261-265.

29. Misra UK, Kalita J, Roy AK, Mandal SK, Srivastava M, 2000. Role of clinical, radiological, and neurophysiological changes in predicting the outcome of tuberculous meningitis: a multivariable analysis. J Neurol Neurosurg Psychiatry 68: 300-303.

30. Misra UK, Kalita J, Srivastava M, Mandal SK, 1996. Prognosis of tuberculous meningitis: a multivariate analysis. $J$ Neurol Sci 137: $57-61$.

31. Modi $\mathrm{M}$ et al., 2017. Clinical and radiological predictors of outcome in tubercular meningitis: a prospective study of 209 patients. Clin Neurol Neurosurg 161: 29-34.

32. Hosoglu $S$ et al., 2002. Predictors of outcome in patients with tuberculous meningitis. Int J Tuberc Lung Dis 6: 64-70.

33. Teasdale G, Jennett $B, 1974$. Assessment of coma and impaired consciousness. A practical scale. Lancet 2: 81-84.

34. Weir CJ, Bradford AP, Lees KR, 2003. The prognostic value of the components of the glasgow coma scale following acute stroke. QJM 96: 67-74.

35. Tsao JW, Hemphill JC 3rd, Johnston SC, Smith WS, Bonovich DC, 2005. Initial glasgow coma scale score predicts outcome following thrombolysis for posterior circulation stroke. Arch Neurol 62: 1126-1129.

36. Ooi MH et al., 2008. The epidemiology, clinical features, and longterm prognosis of Japanese encephalitis in central sarawak, Malaysia, 1997-2005. Clin Infect Dis 47: 458-468.

37. Misra UK, Kalita J, 2002. Prognosis of Japanese encephalitis patients with dystonia compared to those with parkinsonian features only. Postgrad Med J 78: 238-241.

38. Seymour CW et al., 2016. Assessment of clinical criteria for sepsis: for the third international consensus definitions for sepsis and septic shock (sepsis-3). JAMA 315: 762-774.

39. Kalita J, Ranjan A, Misra UK, 2016. Outcome of Guillain-Barre syndrome patients with respiratory paralysis. QJM 109: 319-323.

40. Kalita J, Kohat AK, Misra UK, 2014. Predictors of outcome of myasthenic crisis. Neurol Sci 35: 1109-1114.

41. Netto AB, Taly AB, Kulkarni GB, Shivaji R, 2011. Mortality in mechanically ventilated patients of Guillain-Barré syndrome. Ann Indian Acad Neurol 14: 262-266.

42. Ranjan P, Kalita J, Misra UK, 2003. Serial study of clinical and CT changes in tuberculous meningitis. Neuroradiology 45: 277-282. 